\title{
Psychoacoustic evaluation of musical sounds
}

\author{
ERNST TERHARDT \\ Institute of Electroacoustics, Technical University of München \\ Arcisstrasse 21, D-8000 München 2, West Germany
}

\begin{abstract}
A review is given of several basic aspects of musical sounds, i.e., the perception of duration, the perception of sound impulses as events within temporal patterns, timbre, the equivalence of sensational intervals, roughness, and the pitch qualities of complex sounds. Selected examples illustrate how psychoacoustic results can contribute to the evaluation of musical sounds and to the understanding of the perception of music.
\end{abstract}

Music is a "language" whose acoustic realization usually is prescribed much more precisely and rigidly than is the case with other auditory signals, including speech. In particular, the frequencies of musical tones and the time pattern in which they are realized are essential and significant carriers of information. But the ultimate receiver of this information is the auditory system. It is not the physical sound parameters as such (e.g., frequency and temporal envelope of tones) that are the decisive criteria of musical performance, but the corresponding auditory qualities (e.g., subjective duration, rhythm, timbre, roughness, pitch, harmony). Hence, the quantitative relations between stimulus parameters and auditory sensations are of particular importance in the theory as well as the realization of music. It is particularly in the electronic realization of music, where the physical parameters of musical stimuli in many cases are specified numerically without auditory feedback, that it is desirable to know as precisely as possible how acoustic stimuli are transformed into auditory sensations. Moreover, careful analysis of those psychoacoustic transformations may provide valuable theoretical insight into such basic musical phenomena as, e.g., consonance and harmony.

The present contribution reflects the author's endeavor to proceed into that part of musical perception. Several examples of psychoacoustic evaluation, selected from the author's and his colleqgues' own work, will be discussed. The study may be useful as an introduction into an interesting aspect of psychophysics, a guide to many experimental results, and a stimulus for further research. It is not intended to present a comprehensive review of contemporary results.

The essential content of this contribution was orally presented at the Workshop on Physical and Neuropsychological Foundations of Music, Ossiach/Austria, July 18-22, 1977. The described research of the present author was carried out in the Sonderforschungsbereich Kybernetik, München, supported by the Deutsche Forschungsgemeinschaft. The author is indebted to $\mathrm{H}$. Fastl, who read the manuscript and made valuable suggestions. Also, the helpful comments given by the two referees of Percéption and Psychophysics on a first draft are gratefully acknowledged.

\section{DURATION OF TONES}

A simple parameter of a single musical sound is its duration; corresponding to the physical duration is a psychoacoustic attribute, called subjective duration. The relation between these two parameters is not as simple as may be expected at first sight (cf. Zwicker, 1970). See Figure 1 for an example in some experimental results of Burghardt (1973).

In one experiment, Burghardt presented alternately a $1-\mathrm{kHz}$ tone and a $200-\mathrm{Hz}$ tone in endless succession, and made the subjects adjust the physical duration of one of the tones in such a way that successive tones were perceived as having the same apparent duration. Care was taken to eliminate methodological effects. The relation between the physical durations of the two tones $\left(T_{1 \mathrm{kHz}}\right.$ and $T_{f}$, with $\left.f=200 \mathrm{~Hz}\right)$ under the condition of equal subjective durations is shown by the curve marked $f=200 \mathrm{~Hz}$. The departure of this curve from the dashed line indicates that the $200-\mathrm{Hz}$ tone has to be presented with a distinctly greater physical duration than the $1-\mathrm{kHz}$ tone to be perceived with the same apparent duration.

In another experiment, a $1-\mathrm{kHz}$ tone and a $3,200 \mathrm{~Hz}$ tone were matched in duration in the same way. The result is shown also in Figure 1. Clearly, subjective duration of pure tones depends not only on physical duration, but also on frequency. When the physical duration of a pure tone is held constant, its subjective duration is distinctly diminished by decreasing frequency. It should be noticed that the range of durations in which the effect is significant is almost identical with the range of durations covered by the majority of musical tones (durations of less than about $800 \mathrm{msec}$ ).

With some caution, these results may be applied to real musical tones, which usually are complex tones, i.e., tones composed of several harmonics. Experimental data on the subjective duration of complex tones are not available as yet, but one may infer rather safely that the subjective duration of a complex tone will be determined by its dominant harmonics. For example, it may be predicted that a 


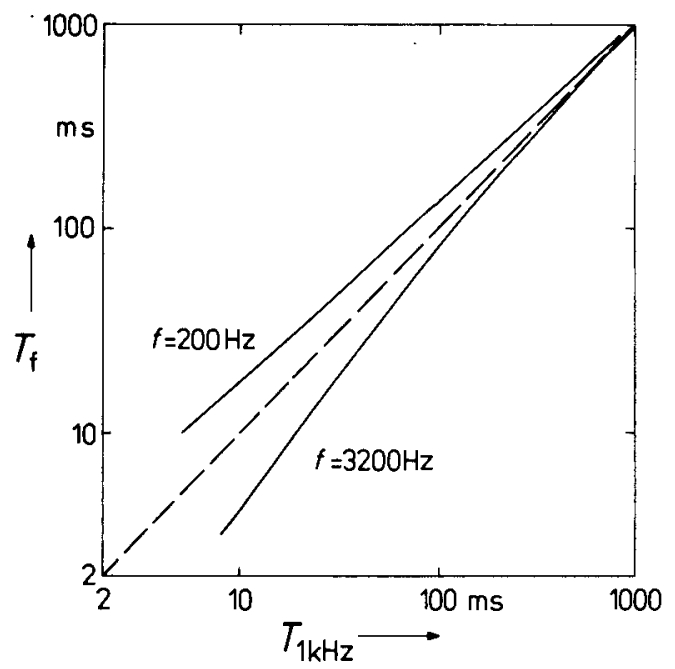

Figure 1. Duration $T_{f}$ of a tone with frequency $f=200$ and $3,200 \mathrm{~Hz}$, respectively, as a function of the duration of $T_{1 \mathrm{kH}}$ of a 1-kHz tone, for equal subjective duration; SPL $60 \mathrm{~dB}$ (from Burghardt, 1973).

complex tone of a certain physical duration, with $200-\mathrm{Hz}$ fundamental frequency, and many strong harmonics up to about the 20th will possess approximately the subjective duration typical of a high pure tone (frequency of some $\mathrm{kHz}$ ) instead of the (shorter) subjective duration typical of a pure $200-\mathrm{Hz}$ tone.

\section{EQUABILITY OF TONE SEQUENCES}

In music, the rhythmic relations between successive tones are an important (and usually carefully prescribed) feature. A tone sequence is defined as being subjectively equable when the successive events which are determined by the single tones are perceived as being uniformly arranged in time. It is usual to consider a tone sequence as "equable" or "uniform" when the physical onsets of the single impulses are arranged equidistantly on the physical time scale. This is an arbitrary definition of equability, which is fairly compatible with common auditory experience. Yet it was considered as sensible to check its psychoacoustic validity (Terhardt \& Schütte, 1976).

Figure 2 illustrates the experimental procedure with two examples $(\mathrm{A}, \mathrm{B})$. A sequence of tones was presented, alternately composed of two tones with different durations and/or temporal envelopes. In case $\mathrm{A}$, both successive tones were rectangularly shaped, but different in duration. In case B, the two tones additionally were different in shape of envelope. The time interval between each pair of identical tones, $T$, was constant. The time interval between the successive (i.e., nonidentical) tones was varied until the criterion of subjective equability was fulfilled. The experiments were carried out with various $T$ values $(0.2 \mathrm{sec} \leqslant \mathrm{T} \leqslant 2 \mathrm{sec})$, combinations of tone dura- tions (5-400 msec), and combinations of envelope shapes; the tone frequency was around $3 \mathrm{kHz}$.

Under the condition of subjective equability, the time interval between the onsets of two successive tones was in many cases not $\mathrm{T} / 2$ as might be expected, but systematically different from this value by a certain departure $\Delta \mathrm{t}$, as indicated and defined in Figure 2. With rectangularly shaped tones of different duration (as in Figure 2A), $\Delta \mathrm{t}$ was maximally found to be $20 \mathrm{msec}$. With tone combinations similar to the example in Figure $2 \mathrm{~B}$, the average $\Delta \mathrm{t}$ value attained about $60 \mathrm{msec}$. It was found that these values were almost independent of $\mathrm{T}$. Moreover, the results were almost independent of sound pressure level and tone (or sound) spectrum (cf. also Schütte, 1978a, 1978b). Similar results, which agree well with these observations, have recently been published by Morton, Marcus, \& Frankish (1976).

\section{TIMBRE OF SLIGHTLY INHARMONIC COMPLEX TONES}

The timbre of a steady sound is to some extent dependent on the spectral envelope (i.e., the gross spectral distribution of intensity), for example the third-octave-band spectrum (cf., e.g., Plomp, Pols, $\&$ Van de Geer, 1967; von Bismarck, 1974). But a considerable part of timbre is not specified by the spectral envelope. Rather, the precise frequencies of the part tones covered by a given envelope also play a role. In electronic organs, for example, synthesis of complex tones by superposition of pure tones which are taken from the equally tempered scale is a usual method. In these synthesized complex tones, only those partials which are one or more octaves above the fundamental are "true" harmonics; the other partials are slightly inharmonic. For example,

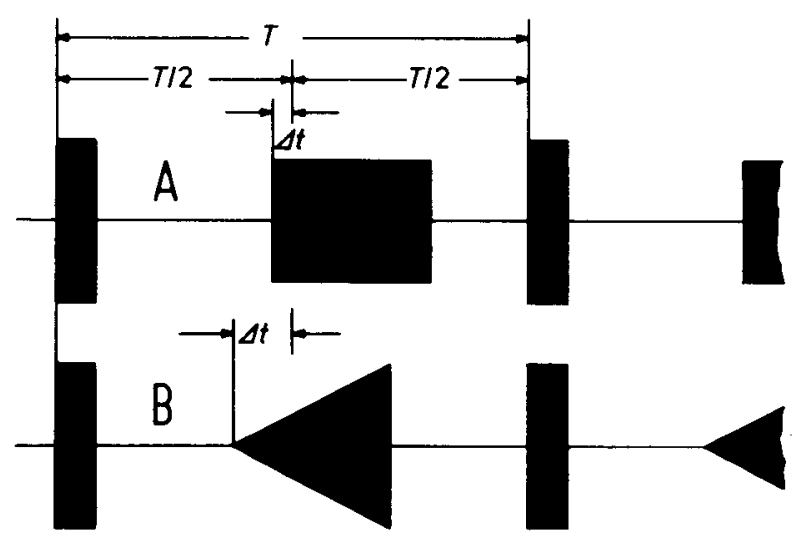

Figure 2. Two examples $(A, B)$ of tone sequences which are perceived as equable (uniform); schematic amplitude-time pattern. The sequences are periodic with period $T$. The departure $\Delta t$ indicates that perceived equability is not identical with equal time intervals between physical onsets. 
the 3rd partial differs from the true 3rd harmonic by -2 cents, the 5 th partial from the true 5 th harmonic by +14 cents. Though the relative amplitude of each partial may be controlled in a wide range, thus providing many distinctly different spectral envelopes, the sound of those electronic organ tones is somewhat uniform and specific. The timbre of a complex tone which is synthesized in the described way is more or less different from that of a truly harmonic complex tone comprising the same number of partials with the same amplitudes.

This difference in timbre was investigated in the following way. A slightly inharmonic complex tone with $220-\mathrm{Hz}$ fundamental frequency, comprising $\mathrm{n}$ partials with equal amplitudes, was synthesized from the equally tempered scale, as described. Moreover, a truly harmonic complex tone with the same fundamental frequency, number, and amplitude of partials was produced by suitable high-pass filtering of a periodic pulse train. These two complex tones (in Figure 3 labeled as stimulus pair " $A$ ") were presented successively in order to be judged on their timbre difference. This timbre difference was measured by means of another pair of stimuli: a pure tone with $220-\mathrm{Hz}$ frequency, followed by a truly harmonic complex tone with $220-\mathrm{Hz}$ fundamental frequency and $\mathrm{m}=1+\Delta \mathrm{m}$ harmonics, where $\Delta \mathrm{m}$ was variable (" $B$ " in Figure 3). By a two-alternative forced-choice method, that number $\Delta \mathrm{m}$ was determined which produced a timbre difference within stimulus pair B which was equivalent to the timbre difference within stimulus pair $\mathrm{A}$. Hence, $\Delta \mathrm{m}$ represents that number of true harmonics which has to be added to a pure tone in order to produce a change of timbre equal to the timbre difference between a slightly inharmonic complex tone and the "same," but truly harmonic, complex tone.

The results are depicted in Figure 3. When $n<3$,

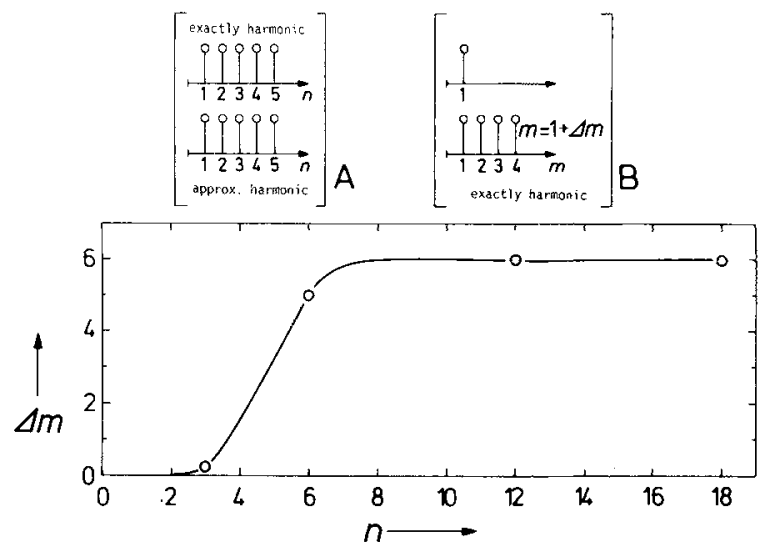

Figure 3. Effect of slight inharmonicity on timbre: Number of truly harmonic overtones which must be added to a pure tone, $\Delta \mathrm{m}$ (stimulus pair B), to produce a timbre difference that is equivalent to that existing between a truly harmonic and a slightly inharmonic complex tone, each comprising $n$ partials with equal amplitudes (stimulus pair $\mathbf{A}$ ). Fundamental frequency of all tones is $220 \mathrm{~Hz} ; 8$ subjects. there is no timbre difference, because both complex tones of pair $\mathbf{A}$ are identical in this case. The inharmonicity effect comes into play when $n=3$. With $n>3$, the difference increases rapidly, attaining saturation.

In electronic organs, there usually is $\mathrm{n} \leqslant 6$. With $\mathrm{n}=6$, the effect of inharmonicity has reached almost its maximally possible magnitude, which is equivalent to the timbre difference between a single pure tone and the same tone "enriched" by six additional true harmonics. This difference may be evaluated as rather considerable. Its evaluation will be enabled more quantitatively and universally by the psychophysical principle described in the following section.

\section{EQUIVALENCE OF SENSORY INTERVALS}

As has been demonstrated by Stevens (1959), in particular, it is feasible to compare the absolute magnitude of one sensory attribute with that of another one, even intermodally. In the same sense, intervals of different sensory attributes may also be judged in terms of their relative width. For example, a subject is able to decide with sufficient confidence whether a particular pitch difference between two successive tones is greater or smaller than a loudness difference existing between the same tones. In this way, that interval of one sensory attribute can be determined which is equivalent to (i.e., neither larger nor smaller than) a given interval of another attribute. With the attributes of pitch, loudness, roughness, and timbre, a simple principle of the equivalence of intervals was found: Intervals along two different sensory continua (attributes) are equivalent to each other when they comprise the same number of just noticeable differences (Terhardt, 1968a). Recently, Suchowerskyj (1977) carried out various experiments on the evaluation of perceptual differences between complex sounds. His results confirm the principle of equivalence. Moreover, he demonstrated that on the basis of this principle the perceived distance (i.e., dissimilarity) between complex stimuli may be described concisely: The perceived distance corresponds to the distance within a Euclidian space in which the stimuli are represented by points. The dimensions of that space are the involved attributes, scaled in just noticeable differences.

It should be noted that the observed relation between equivalent sensory intervals and just noticeable differences is,neither identical with Fechner's (1860) law nor in conflict with Stevens' (1957) "power law." Both Fechner's and Stevens' laws are pertinent to the relation between the absolute magnitude of a sensory attribute and the corresponding stimulus parameter, whereas the principle of equivalence reflects the evaluation of intervals of different attributes relative to each other. 

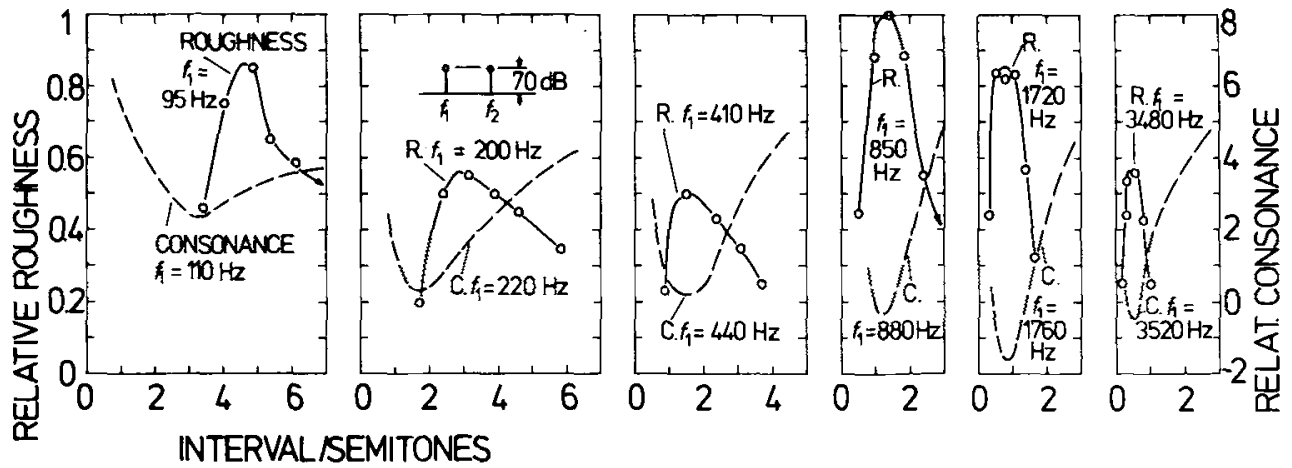

Figure 4. Roughness (solid curves, left ordinate) and musical consonance (dashed curves, right ordinate; after Kameoka \& Kuriyagawa, 1969) of pure-tone pairs, as a function of interval, in six different frequency regions as indicated. Interval width is expressed in semitones, i.e., interval $=12 \operatorname{ld}\left(f_{2} / f_{1}\right)$ semitones (from Terhardt, 1977).

Due to its simplicity, the principle of equivalence may be quite helpful in the psychoacoustic evaluation of musical sounds. By means of this principle, a given perceptual difference that is to be evaluated can be expressed by another perceptual difference with which one is more familiar. For example, it was found that the maximal timbre difference between an approximately harmonic and a truly harmonic complex tone, as discussed in the foregoing section (Figure 3), comprises about 15 just noticeable differences. Hence, this timbre difference is approximately equivalent to the pitch step $\mathrm{C}_{5}-\mathrm{D}_{5}$ of a musical tone, because this step also comprises about 15 just noticeable differences. Another equivalent sensory interval is the loudness difference between two broadband noises differing in sound pressure level by about $15 \mathrm{~dB}$ (because the just noticeable difference of that noise is about $1 \mathrm{~dB}$ ).

\section{ROUGHNESS}

Roughness is a sensory attribute which is produced by amplitude fluctuations of the auditory excitation, e.g., beats. It has a strong influence on the evaluation of musical sounds. In most cases, roughness is undesired, as it diminishes the degree of consonance.

With sinusoidally amplitude-modulated tones, it has been found that the perceived magnitude of roughness is approximately proportional to the square of the degree of modulation (Terhardt, 1968b). Other basic principles of the transformation of sound parameters into roughness may be studied in experiments with dyads of pure tones (two-tone complexes). Figure 4 depicts how the roughness of a pure-tone dyad depends on the frequency interval and the frequency region in which the dyad is situated (from Terhardt, 1977). The roughness magnitudes depicted by this diagram were obtained by adjusting the degree of modulation of a sinusoidally amplitudemodulated pure tone with constant carrier and modulation frequency in such a way that the perceived roughness of that $\mathrm{AM}$ tone was equal to that of the pure-tone dyad. It is evident from the diagram that, with increasing frequency interval (i.e., increasing beat frequency), roughness grows up to a certain maximum and vanishes for sufficiently large intervals.

The roughness of a complex tone stimulus, comprising many partials (as does, e.g., a musical chord), may be considered with some approximation to be composed of partial roughnesses which are produced by pairs of adjacent partials. Figure 5 illustrates this principle with a simple model (cf. Terhardt, 1974a).
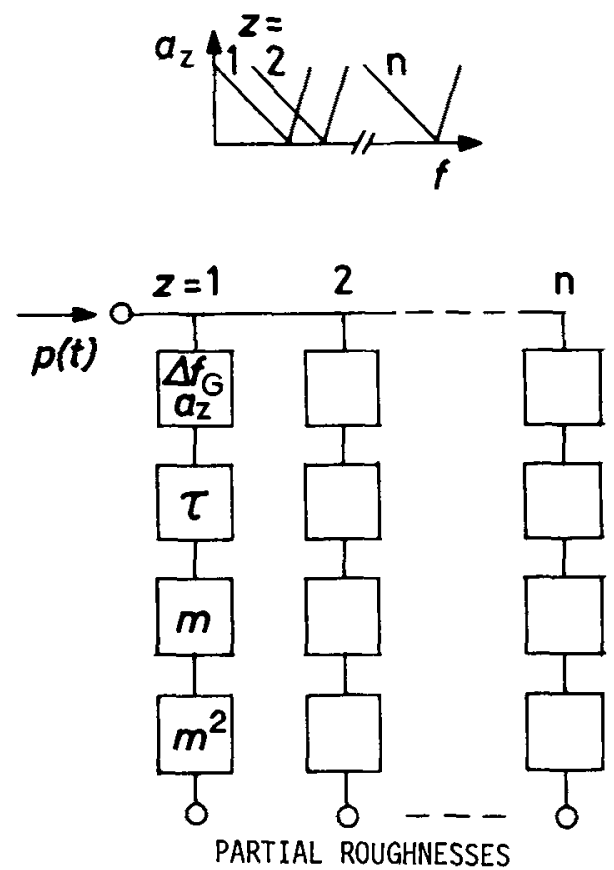

Figure 5. Simple functional model of roughness perception. $p(t)=$ sound pressure (signal) input; $z=$ number of critical-band filter, frequency response of the filters is schematically shown on top $\left[a_{2}(f)\right] ; \Delta f_{G}=$ critical bandwidth; $\tau=$ time constant of envelope detection, equal in all channels; $m=$ degree of modulation (relative amplifude fluctuation). Roughness of complex sounds is composed of partial roughnesses, produced in the adjacent channels. 
According to this model, roughness is composed of the partial roughnesses occurring in the critical bands of the auditory system. As a general rule, it can be said that a stimulus which does not produce envelope fluctuations in any critical band does not produce roughness. This is the case when the partials of a complex stimulus are spaced by frequency distances exceeding the critical bandwidth. An additional limitation of roughness perception is the auditory system's low-pass characteristic with respect to the rate of envelope fluctuations. This is reflected in the model by the time constant $\tau$, which is of the order of $10 \mathrm{msec}$. The result is that stimulus fluctuations exceeding a rate of about $300 \mathrm{~Hz}$ do not produce any roughness, even if the spectral components fall within the same critical band. This applies, for example, to single harmonic complex tones, whose partials (harmonics) have a frequency separation equal to the fundamental frequency; a harmonic complex tone with a fundamental frequency higher than about $300 \mathrm{~Hz}$ (approximately the note $\mathrm{D}_{4}$ ) will in any case be free from roughness.

When two or more complex tones are superimposed, roughness may be produced also by the beats of adjacent partials stemming from different complex tones. Consequently, the roughness in this case depends on the relative fundamental frequencies of the complex tones as well as on their overtone structure. In particular, when the fundamental frequencies of simultaneous harmonic complex tones are in a ratio of small integer numbers, roughness will attain a certain minimum. This principle was discovered by Helmholtz (1863), who considered it as a basic feature of musical consonance. Experiments on musical consonance by Kameoka and Kuriyagawa (1969) and Plomp and Levelt (1965) fully confirm Helmholtz's concept, demonstrating that, with isolated, single stimuli (i.e., chords), roughness is the decisive factor which governs musical consonance. In Figure 4, the results of Kameoka and Kuriyagawa on the evaluation of consonance, obtained with puretone dyads, are depicted by the dashed curves. Clearly, consonance is the "inverse" of roughness. It should be noted, however, that this close relation between roughness and consonance is pertinent to single, isolated sound stimuli. Such other important features of musical sounds as tonal affinity and harmony, which may also be considered as being a part of musical consonance, are not explained by this relation. It was concluded, therefore, that musical consonance (in a general sense) may be composed of two main components, sensory consonance and harmony (Terhardt, 1976, 1977), and that, in the evaluation of musical consonance of isolated stimuli, only the first component (sensory consonance) is apparent, because it is dominating in such an experimental condition. Some possible foundations of the second component, harmony, will be discussed in the following sections.

\section{PITCH OF COMPLEX TONES}

The physical parameter which represents the particular musical note played in each moment, is the fundamental frequency of the corresponding tone. The sensory attribute which specifies the note is pitch. Musical tones in most cases are harmonic complex tones, and the pitch sensation corresponding to their fundamental frequency is established by the auditory system even in cases where the fundamental as a spectral component is weak or absent (cf. Schouten, Ritsma, \& Cardozo, 1962). Since a sinusoidal spectral component, i.e., a pure tone, also produces a pitch which rather directly is determined by its frequency, it is advisable to make a distinction between two kinds of pitch: spectral pitch, i.e., the pitch produced by a pure tone, and virtual pitch, i.e., the pitch assigned to the entire complex tone.

The psychoacoustics of spectral-pitch perception still bears many open questions. Yet there are many hints that spectral pitch is closely related to the place of maximal excitation of the organ of Corti within the cochlea. The perception of virtual pitch presently is preferably considered as a process of auditory pattern recognition (Goldstein, 1973; Terhardt, 1970, 1972, 1974b; Wightman, 1973). With respect to the psychoacoustic evaluation of musical sounds, it may be useful to depict the psychophysical nrinciples of this process and some consequences thereof (the following description is pertinent to the author's model).

The principles that are presumed to be valid in that perceptual process are largely identical with established and rather obvious features of highly developed sensory systems. They may be listed as follows.

(1) A higher sensory system is able to discriminate certain elementary stimuli of which a stimulus pattern is composed. Thus it is presumed that the auditory system is able to resolve several partials of a complex tone, and to perceive them separately. This presumption is justified by the finding that the spectral pitches of certain partials of a complex tone may be consciously perceived (Helmholtz, 1863; Plomp, 1964). Of course, other elementary' cues, such as temporal fluctuations and timbre components, may be detected also.

(2) When a particular type of stimulus pattern (i.e., a specific arrangement of elements) does occur repeatedly, a higher sensory system is able to recognize and memorize that specific arrangement. Thus, it is presumed that the auditory system is able to recognize and memorize the spectral-pitch intervals existing between those partials which normally are resolved. For physical reasons, a highly specific 
arrangement of partials is realized in periodic signals (i.e., harmonic complex tones): Each periodic signal with a given period (i.e., fundamental frequency) possesses one and only one arrangement of partials; each fundamental frequency corresponds to one specific arrangement. In normal human life, certain periodic signals with various fundamental frequencies are of highest biological relevance and occur frequently: the voiced sounds of speech. Hence, the chance that the auditory system normally will memorize the specific arrangement of harmonic partials is high. Of course, the auditory system, while developing the ability to identify the sounds of a particular language, memorizes many more cues relevant to speech perception; but these are not essential in the present consideration of pitch perception.

(3) When a higher sensory system has memorized a specific stimulus arrangement, this particular arrangement, if presented again, will be perceived as one entity with a specific label, rather than a meaningless set of elements. Thus, it is presumed that the auditory system, being already familiar with the specific arrangement of the spectral pitches of a harmonic complex tone, will perceive that tone as an entity which is labeled by a specific virtual pitch.

(4) When a higher sensory system has developed the ability to perceive a pattern as an entity, the identification and labeling of that pattern will not break down when some of its elements are absent, provided that the remaining elements are objectively sufficient to specify the pattern. Thus, it is presumed that the auditory system is able to label a given complex tone by its proper virtual pitch even in cases where some partials, the fundamental included, are absent. This actually is observed as a basic feature of virtual pitch ("residue pitch").

(5) When a higher sensory system has developed the ability to identify patterns by means of a reduced number of elements, it may develop the habit to identify even a complete pattern, preferably by means of only a restricted ensemble of all the elements available. Thus, it is presumed that the auditory system deduces the virtual pitch of a complex tone preferably from those partials which lie in a frequency region where the system itself is working optimally and where essential and frequently occurring stimuli bear a maximum of tonal information. This applies to the frequency region of about 500 to $1,500 \mathrm{~Hz}$, where the auditory threshold is low, the just noticeable difference of frequency is small, and speech bears its essential tonal information (most of the first and second formants of vowels lie in that region). The existence of that dominant frequency region has, in fact, been experimentally demonstrated (Plomp, 1967; Ritsma, 1967).

Since the spectral-pitch patterns established by complex tones are one-dimensional, i.e., relatively simple, it was possible to incorporate these principles into a model which enables quantitative predictions (Terhardt, 1972, 1974b). In Figure 6, some essential functional relations and quantitative effects are illustrated. As an example, a harmonic complex tone (the note $\mathrm{A}_{3}$, with $220-\mathrm{Hz}$ fundamental frequency) is represented by its amplitude spectrum, comprising 10 harmonics. Also shown is the corresponding pattern of spectral pitches. For the sake of simplicity, all pitch values (i.e., sensory quantities) are represented by corresponding frequencies (i.e., physical quantities), namely by the frequency of a single, unaffected pure tone which produces the same pitch. As may be recognized, the spectral pitch of a particular harmonic is not exactly equal to the pitch of a single pure tone with the same frequency. Rather, certain pitch shifts occur (the fundamental being shifted downwards, the higher harmonics upwards),

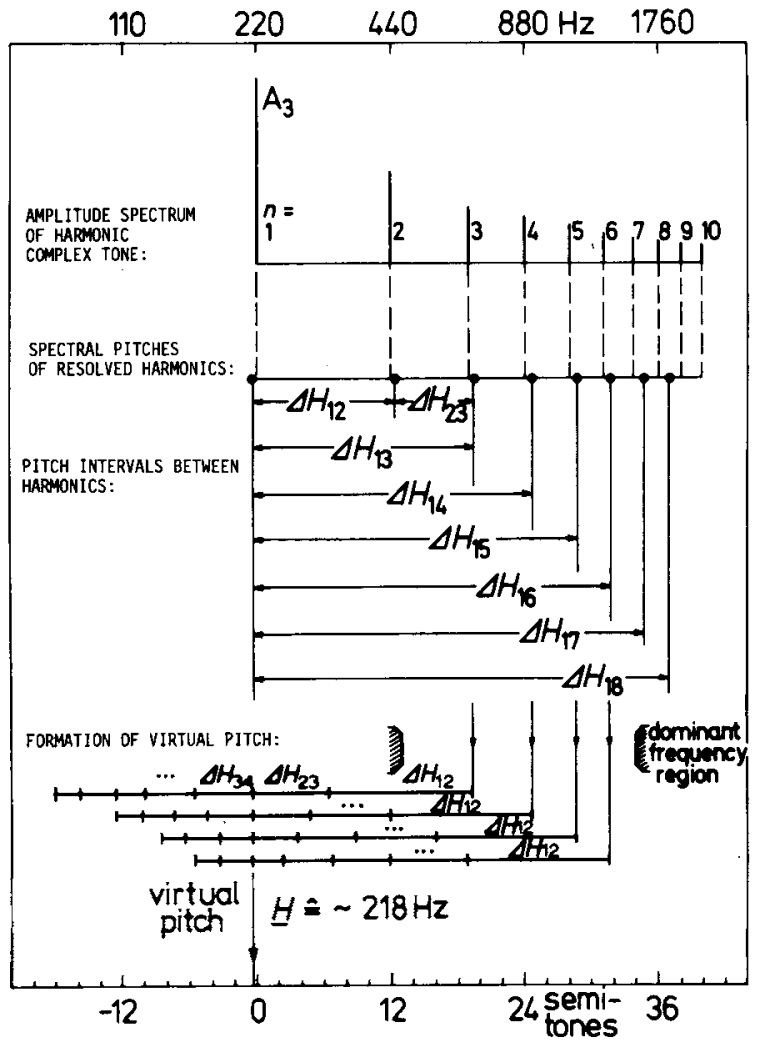

Figure 6. Schematic illustration of the formation of virtual pitch. The lower harmonics of a complex tone (the note $A_{3}$, as an arbitrary example shown with 10 harmonics) produce a pattern of spectral pitches (dots). The pitch values are represented by the frequency values of a single, unaffected pure tone producing the same pitch. The pattern of spectral pitches is memorized by the system and thereafter employed to assign subharmonic virtual pitch to any tonal complex (lower part). Due to mutual interaction between the partials, the spectral pitches of a complex tone usually are slightly shifted in the indicated directions. Thus, the pitch intervals $\Delta H_{12}, \Delta H_{23}$, etc., on the memorized "yardstick" are "stretched." The semitone scale (lower abscissa) is a logarithmic equivalent of the frequency scale (upper abscissa), with 0 semitones corresponding to $220 \mathrm{~Hz}$. 
which may be considered as the result of mutual nonlinear interaction of the adjacent harmonics in the peripheral auditory system. The real existence of the pitch shifts has been experimentally demonstrated (Terhardt, 1971a). They change the pattern of spectral pitches of a complex tone in a specific way: The pitch intervals between the adjacent harmonics, labeled in Figure 6 as $\Delta \mathrm{H}_{12}, \Delta \mathrm{H}_{23}$, etc., are somewhat "stretched" in the sense that a single, unaffected pure tone, matched in pitch to the spectral pitches of those harmonics, will no longer establish a series of harmonic frequencies but rather frequencies whose ratio slightly exceeds the values $1: 2,2: 3$, etc.

Following the principles listed before, it is presumed that the auditory system gets acquainted with that spectral-pitch pattern by repeated and frequent perception of speech. (In fact, essential features of voiced speech sounds are identical with those of the complex tone depicted in Figure 6.) When this phase of "learning" is accomplished, the memorized pattern is unchangeable and is employed to find the proper pitch label of any complex tone perceived thereafter. By means of Figure 6, the deduction of virtual pitch may be illustrated schematically in the following way.

When a complex tone is present, the system inspects each of the partials lying in the dominant frequency region $(500-1,500 \mathrm{~Hz})$ with respect to the question, "Which harmonic of what fundamental may this be?" The answer is obtained by using the memorized pattern of spectral pitches as a "yardstick." This yardstick is shifted horizontally along the actual spectralpitch pattern, and each of its scale marks is successively matched to the inspected partial's spectral pitch. With every match, the horizontal position of the yardstick's origin indicates a certain candidate of the unknown fundamental pitch (virtual pitch). The series of potential fundamental pitches obtained in this way may be represented by another "yardstick," which is identical to the memorized one, but reversed in direction and matched with its origin to the inspected partial's pitch. By inspecting each of the spectral pitches of the dominant region in this way, many potential fundamental pitches are obtained, some of which may coincide within a just noticeable difference, as indicated in Figure 6 by four "reversed yardsticks" which are matched with their origins to the actual spectral pitches of the dominant region. Finally, the pitch value, indicated by the majority of coinciding potential values, is labeled as the virtual pitch of the complex tone. ${ }^{1}$

In the example shown in Figure 6, the resulting virtual pitch is equal to the spectral pitch of the fundamental, corresponding to a frequency of about $218 \mathrm{~Hz}$. The slight departure of this value from $220 \mathrm{~Hz}$ (the actual fundamental frequency) indicates that the virtual pitch of that complex tone is slightly lower than the pitch of a pure tone with the frequency $220 \mathrm{~Hz}$.
Since, in the example discussed before, the fundamental of the complex-tone spectrum was not lying in the dominant region, it was not involved in the formation of virtual pitch. Thus, it is evident that the procedure, in principle, is independent of the actual presence of the fundamental. Figure 7 illustrates the formation of virtual pitch when harmonics 1 and 2 are omitted from the spectrum. It is presumed that the memorized pitch pattern employed by the system is exactly the same as that described before, and also that the procedure is the same. Yet the resulting virtual pitch in this case is slightly lower than before (corresponding to a frequency of $215 \mathrm{~Hz}$ ). This is caused by the fact that the actual pattern of spectral pitches, from which virtual pitch is deduced, is not exactly equal to that shown in Figure 6: The "sharpening" of the 3rd and 4th harmonics, which is produced by the presence of the 1 st and 2 nd harmonics, is now absent or at least reduced, because these two harmonics have been omitted.

Figure 8 provides an outline of the departure of virtual pitch of harmonic complex tones from the pitch of an unaffected pure tone with the same frequency, based on experimental results of Terhardt (1971b) and Walliser (1969a). The curves indicate that, in fact, a complex tone comprising all lower harmonics (as does the one depicted in Figure 6) usually is slightly lower in pitch than a pure tone with the same frequency, and a "residue tone," i.e., a complex tone with lower harmonics being suppressed up to a certain cutoff frequency (as in the one depicted in Figure 7), is even lower. Also shown is how the effect depends on fundamental frequency.

It is obvious that pitch departures of this kind and magnitude may be quite significant in music. Evaluation of musical sounds in terms of pitch will hardly

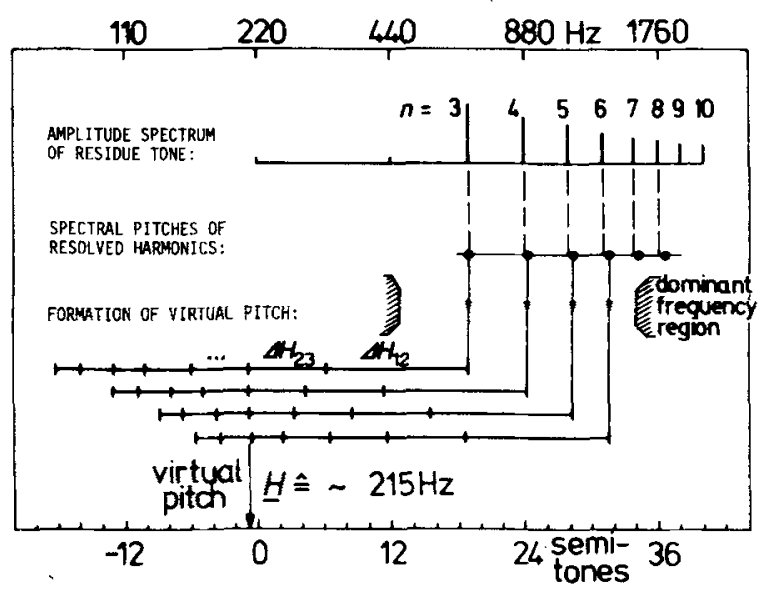

Figure 7. Formation of virtual pitch of a harmonic residue tone (the same tone as in Figure 6, but with the first two harmonics suppressed). The "'yardstick," i.e., the memorized pattern of spectral pitches, is identical with that of Figure 6. Notice that the pitch shift of the 3rd harmonic (the sharpening of its actual spectral pitch) is distincly less than in case of the complete complex tone depicted in Figure 6. 


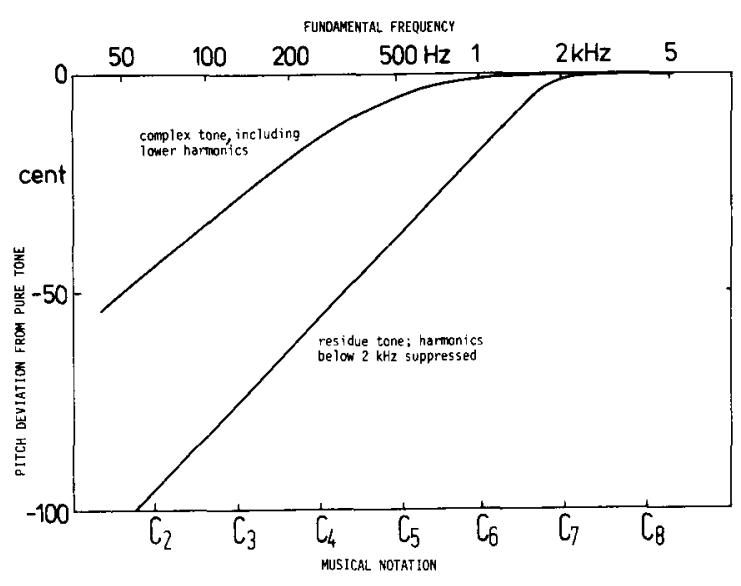

Figure 8. Departure of the virtual pitch of complex tones from the pitch of a pure tone whose frequency is equal to the fundamental frequency, as a function of (fundamental) frequency or musical notation, respectively. 100 cents are identical with 1 semitone, corresponding to a relative frequency difference of almost $6 \%$. The negative values of the departure indicate that complex tones are perceived with a lower pitch than pure tones having the same frequency (from Terhardt, 1971b).

be appropriate without taking these phenomena into account. This applies, for example, to the pitch of a single, slightly stiff, oscillating string, such as a piano string. The effect of the slight inharmonicity of its partials on its virtual pitch is almost negligible in view of the pitch shifts produced by the ear itself.

\section{THE SENSE OF HARMONIC INTERVALS}

It is well known that even listeners without any musical training possess a sense of basic musical intervals, i.e., the octave and the fifth. The principles of virtual pitch perception as described in the foregoing section provide a possible explanation of the origin of that sense: The sense of harmonic intervals appears to be a by-product of speech perception. Memorizing the spectral-pitch patterns of voiced speech sounds (i.e., harmonic complex tones), in order to assign virtual pitch to them as described, is identical to acquiring a sense for the close affinity existing between tones which are an octave, a fifth, etc., apart.

A phenomenon which provides strong support to this explanation is that of octave enlargement. As demonstrated by Corso (1954), Walliser (1969b), Ward (1954), and others, the frequency ratio of two successive tones, adjusted auditively to establish an optimal octave leap, is systematically and consistently greater than 2:1. Since two successive, and thus unaffected, tones provide a direct measure of pitch, as used in the foregoing discussion, this phenomenon directly confirms that the memorized octave interval is "stretched," as may be expected from the suggested explanation of its origin. Of course, while this is not a stringent proof, it does at least provide strong support.

\section{THE FUNDAMENTAL NOTES ("ROOTS") OF MUSICAL CHORDS}

It may be expected that after the auditory system has developed the perceptual faculties to label harmonic complex tones by virtual pitches, it will also do so (or at least attempt to do so) when any arbitrary and perhaps unnatural stimulus is presented. This actually is the case. For example, it has been demonstrated that the procedure of deducing virtual pitch from dominant partials, as described before, is maintained when inharmonic tone complexes are presented (Terhardt, 1972; cf. de Boer, 1956; Walliser, 1969a). By means of an appropriate experimental procedure, it even is possible to make the auditory system produce virtual pitches when, instead of a pattern of partials, just one single pure tone is presented (Houtgast, 1976). The virtual pitches assigned to that pure tone correspond to its subharmonics, as would be predicted by the described procedure, i.e., the "reversed yardstick" indicating potential virtual pitches. Thus, it may be expected that the procedure is also maintained when musical chords, i.e., stimuli which are composed of several harmonic complex tones, are presented. In Figure 9, the same schematic deduction of virtual pitch as described before is applied to a major triad (the notes $A_{4}, D_{5}$, $\mathrm{F}_{5}{ }^{*}$ ). Every part-tone which may be.considered as being resolved by the peripheral auditory system (i.e., not masked by adjacent ones) is considered as a cue to virtual pitch. Several virtual-pitch labels are obtained, namely, the pitches of the tones which actually are present, and in addition certain lower virtual pitches, which are called "fundamental notes."

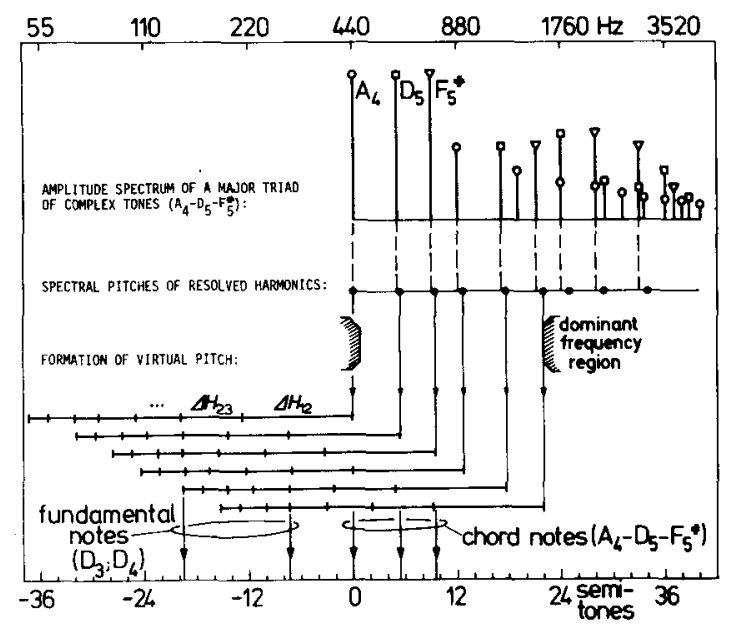

Figure 9. Formation of virtual pitch of a major triad that is composed of harmonic complex tones. The amplitude spectrum was arbitrarily constructed by in-phase superposition of three harmonic complex-tone spectra (the notes $A_{4}, D_{5}, F_{5}^{*}$ ). Application of the same principles as in Figures 6 and 7 yields the pitches of the chord notes $\left(A_{4}, D_{5}, F_{5}{ }^{*}\right)$ and certain fundamental notes $\left(D_{3}, D_{4}\right)$. The latter represent the chord's "root." 


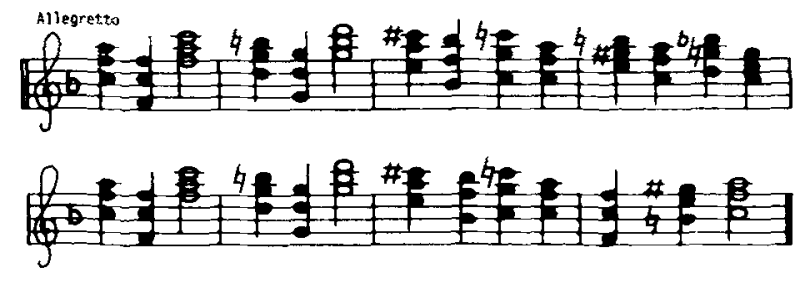

Figure 10 . The sequence of fundamental notes specified by this chord sequence represents a well-known tune. By playing the chord sequence softly on an electronic organ or piano, the tune can be identified.

The fundamental notes are identical in height with those fundamental notes that are assigned by conventional musical theory to that major triad. Also for other types and inversions of musical chords, the fundamental notes which are known from musical theory are predicted by the virtual-pitch model (cf. Terhardt, 1976).

Hence, a new and promising approach to the understanding of the harmonic aspect of musical chords is obtained, which may be depicted as follows. Musical experience and (as a consequence) musical theory tell us that the auditory system usually assigns to a given musical chord a certain label, which depends on the frequency relations between the tones of which the chord is composed. This label is called the chord's "root" or "harmony"; it is represented by the chord's fundamental note ("basse fondamentale"). Apparently, this label is virtually identical with the virtual-pitch label which the auditory system assigns more or less distinctly to any appropriate sound stimulus. Not only with "natural" stimuli (such as voiced speech sounds), but also in the case of being presented with a musical chord, the auditory system appears to inspect the dominant spectral components with respect to the question, "Which harmonics of what fundamental may these be?" If there is a sufficiently unambiguous answer, the chord is perceived as "harmonic," possessing a typical "root." If the answer is too ambiguous, the chord is "disharmonic," having no "root."

Yet this explanation is somewhat theoretical in nature. Therefore, the question of whether the fundamental notes of chords may be really perceived as virtual pitches was investigated. Short sequences of different chords, thus sequences of fundamental notes, were presented, and subjects were asked to identify and write down the fundamental-note melodies they heard. The resulting rate of correctly identified sequences (on the average $48.2 \%$, while the a priori chance was $0.8 \%$ ) indicates a high degree of fundamental-note recognition (Terhardt, 1977). Hence, the fundamental notes may, in fact, be considered as an auditory percept rather than just a theoretical tool.

The perception of fundamental notes may provisionally be demonstrated with the chord sequence shown in Figure 10. The corresponding fundamentalnote sequence represents a well-known tune. This tune is easily identified by most listeners when the sequence is played softly on an electronic organ or piano.

The extremely valuable concept of the fundamental note appears to have been introduced into musical theory in the 18th Century by the French composer and musicologist, Rameau. One may conclude now that he found that concept not merely by intuition, but rather by an ingenious faculty to realize what he actually perceived.

\section{REFERENCES}

BismaRCK, G. von. Timbre of steady sounds: A factorial investigation of its verbal attributes. Acustica, 1974, 30, 146-159.

BuRghARDT, H. Die subjektive Dauer schmalbandiger Schalle bei verschiedenen Frequenzlagen. Acustica, 1973, 28, 278-284.

Corso, J. F. Scale position and performed melodic octaves. Journat of Psychology, 1954, 37, 297-305.

$\mathrm{DE}$ BOER, E. On the "residue" in hearing. Thesis, University of Amsterdam, 1956.

Fechner, G. T. Elemente der Psychophysik. Leipzig: Breitkopf \& Härtel, 1860 .

Golostein, J. L. An optimum processor theory for the central formation of the pitch of complex tones. Journal of the Acoustical Society of America, 1973, 54, 1496-1516.

Helmoltz, H. L. F. voN. Die Lehre von den Tonempfindungen als physiologische Grundlage für die Theorie der Musik. Braunschweig: F. Vieweg, 1863.

Houtgast, T. Subharmonic pitches of a pure tone at low $S / N$ ratio. Joumal of the Acoustical Society of America, 1976, 60, 405-409.

Kameoka, A., \& Kuriyagawa, M. Consonance theory, Part I, II. Joumal of the Acoustical Society of America, 199, 45, $1451 \cdot 1469$.

Morton, J., Marcus, S., \& Frankish, C. Perceptual centers. Psychological Review, 1976, 83, 405-408.

Plomp, R. The ear as a frequency analyzer. Journal of the Acoustical Society of America, 1964, 36, 1628-1636.

Plomp, R. Pitch of complex tones. Journal of the Acoustical Society of America, 1967, 41. 1526-1533.

Plomp, R., \& Levelt, W. J. M. Tonal consonance and critical bandwidth. Joumal of the Acoustical Society of America, 1965, 38, 548-560.

Plomp, R., Pols, L. C. W., \& van de Gegr, J. P. Dimensional analysis of vowel spectra. Journal of the Acoustical Society of America, 1967, 41. 707-712.

RITsMs, R. J. Frequencies dominant in the perception of the pitch of complex sounds. Journal of the Acoustical Society of America, 1967, 42, 191-198.

Schouten, J. F., Ritsma, R. J., \& Cardozo, B. L. Pitch of the residue. Joumal of the Acoustical Society of America, $19 \% 2$, 34, 1418-1424.

SCHüTrE, H. Subjektiv gleichmässiger Rhythmus. Ein Beitrag zur zeitlichen Wahrnehmung von Schallereignissen. Acustica, in press. (a)

SCHürTe, H. Ein Funktionsschema für die Wahrnehmung eines gleichmässigen Rhythmus in Schallimpulsfolgen. Biological Cybernetics, in press. (b)

Stevens, S. S. On the psychophysical law. Psychological Review, 1957. 64, 153-181.

Stevens, S. S. Cross-modality validation of subjective scales for loudness, vibration and electric shock. Joumal of Experimental Psychology, 1959, 57, 201-209.

Sucrowersky, $W$. Beurteilung von Unterschieden zwischen aufein anderfolgenden Schallen. Acustica, 1977, 38, 131-139.

TERHARDT, E. Über ein Aquivalenzgesetz für Intervalle akustischer 
Empfindungsgrössen. Biological Cybermetics (Kybernetik), 1968 5, 127-133. (a)

TERHARDT, E. Über akustische Rauhigkeit und Schwankungsstärke. Acustica, 1968, 20, 215-224. (b)

TERHARDT, E. Frequency analysis and periodicity detection in the sensations of roughness and periodicity pitch. In R. Plomp \& G. F. Smoorenburg (Eds.), Frequency analysis and periodicity detection in hearing. Leiden (Netherlands): Sijthoff, 1970. Pp. 278-287.

Terhardt, E. Pitch shifts of harmonics, an explanation of the octave enlargement phenomenon. Proceedings of the 7 th International Congress of Acoustics (Budapest), 1971, 3, 621-624. (a)

TerhardT, E. Die Tonhöhe harmonischer Klänge und das Oktavintervall. Acustica, 1971, 24, 126-136. (b)

TERHARDT, E. Zur Tonhöhenwahrnehmung von Klängen, I, II Acustica, 1972, 26, 173-199.

TERHARDT, E. On the perception of periodic sound fluctuations (roughness). Acustica, 1974, 30, 201-213. (a)

TERHARDT, E. Pitch, consonance, and harmony. Journal of the Acoustical Society of America, 1974, 55, 1061-1069. (b)

TERHARDT, E. Ein psychoakustisch begründetes Konzept der Musikalischen Konsonanz. Acustica, 1976, 36, 121-137.

TERHARDT, E. The two-component theory of musical consonance. In E. F. Evans \& J. P. Wilson (Eds.), Psychophysics and physiology of hearing. London: Academic Press, 1977. Pp. 381-390.
Terhardt, E., \& SchütTE, H. Akustische Rhythmus-Wahrnehmung: Subjektive Gleichmässigkeit. Acustica, 1976, 35, 122-126.

WaLLISER, K. Zusammenhänge zwischen dem Schallreiz und der Periodentonhöhe. Acustica, 1969, 21, 319-329. (a)

W ALLISER, K. Über die Spreizung von empfundenen Intervallen gegenuber mathematisch harmonischen Intervallen bein Sinustönen. Frequenz, 1969, 23, 139-143. (b)

WARD, W. D. Subjective musical pitch. Journal of the Acoustical Society of America, 1954, 26, 369-380.

Wightman, F. L. The pattern-transformation model of pitch. Journal of the Acoustical Society of America, 1973, 54, 407-416.

Zwicker, E. Subjektive und objektive Dauer von Schallimpulsen und Schallpausen. Acustica, 1970, 22, 214-218.

\section{NOTE}

1. In many applications, the pitch shifts may be disregarded. Then the frequency corresponding to virtual pitch may be obtained simply by determining the common integer fraction of the frequencies of the dominant partials.

(Received for publication August 29, 1977; revision accepted February 20, 1978.) 